\title{
Effects on tolerance of chimerism and graft-versus-host disease in vascularized bone marrow allotransplantation
}

\author{
Jong Won Hong ${ }^{1}$, Jung Hyun Lim² \\ ${ }^{1}$ Department of Plastic and Reconstructive Surgery, Severance Hospital, Seoul, Korea \\ ${ }^{2}$ Institute for Human Tissue Restoration, Yonsei University College of Medicine, Seoul, Korea
}

Background: Various studies have been performed on immune tolerance in vascularized composite tissue allotransplantation (VCA). Typical researches were the induction of immune tolerance using stem cells. However, the survival and influence period of externally injected stem cells were not so much long. We tried to find out how much immune tolerance occurs through vascularized bone marrow allotransplantation based on the femoral bone flap.

Methods: Vascularized bone marrow allotransplantation was performed. Donor used a 7-8-week-old Brown Norway rat (BN, RTn). Recipients were 7-8-week-old Lewis rats (LEW, RT11). Femoral bones were used for immune tolerance materials for living stem cells. Immunosuppression was given daily intraperitoneally with FK-506. For chimerism analysis, BN rat antigen in LEW rat blood was investigated. Tregs were measured for the role of immune tolerance. In addition, T cells, B cells, helper T (Th), cytotoxic T (Tc), and natural killer (NK) cells were analyzed. Mixed lymphocyte reaction was performed.

Results: It was difficult to conclude of changes in T cells, B cells, Th, and Tc in the individuals who survived for 8 weeks. However, Treg showed an overall increase. NK cells showed a similar pattern to Th change. Chimerism was most seen in the first week and maintained similarly from the second week. When compared with the deceased rats below 2 weeks, the increase in NK cells and Tregs was rather high.

Conclusions: When vascularized bone marrow allotransplantation was performed, there was an effect of chimerism. However, there was a limit to explaining the effect of immune tolerance with only the change in Tregs compared to those who died earlier. Further studies are needed to conclude that it increases the survival of transplants in previous many reports.

Corresponding author: Jong Won Hong

E-mail: hsaturn@hanmail.net

(c) The Korean Society for Transplantation

This is an Open Access article distributed under the terms of the Creative Commons Attribution Non-Commercial License (http://creativecommons.org/licenses/by-nc/4.0/) which permits unrestricted non-commercial use, distribution, and reproduction in any medium, provided the original work is properly cited. 